\title{
Performance Assessment of Distance Protection in Systems with High Penetration of PVs
}

\author{
Alexander Novikov, Jose de Jesus Chavez and Marjan Popov \\ Faculty of EEMCS \\ Delft University of Technology \\ Delft, The Netherlands \\ A.Novikov@student.tudelft.nl, J.J.ChavezMuro@tudelft.nl, M.Popov@tudelft.nl
}

\begin{abstract}
This paper deals with the investigation of the performance of distance protection in a $400 \mathrm{kV}$ transmission line for a system with high penetration of photovoltaic sources (PV). A grid with a PV plant is designed in RSCAD software environment, which is supported by RTDS. By performing hardware-in-the-loop tests, two commercial relays are investigated in real-time. Firstly, the distance protection function is tested with a synchronous generator to check the response of the studied system and validate correct relay settings. Afterwards, the synchronous generator is replaced by a generic PV plant model. The impact of different fault locations, fault types and fault impedances are studied. Finally, the critical scenarios in the power system are determined for which a relay fails to detect a fault. Many simulations are performed and cases, when a relay unexpected operations, are identified. Relevant solutions to overcome these problems are suggested.
\end{abstract}

Index Terms-- Distance protection function, Hardware-in-theloop test, Photovoltaic plant, Real-time digital simulation, VSC converter.

\section{INTRODUCTION}

In compliance with the Paris Agreement, many countries stimulate the increase of the renewable energy generation in the power systems. According to European plans on climate change [1], in the European Union, $20 \%$ of the energy should be produced by renewables by 2020 . In Australia, based on "Emissions Reduction Target" [2], the renewable energy target allows sustainable growth for both small and large scale renewable technologies to produce more than $23 \%$ of Australia's electricity from renewable sources by 2020. In order to achieve higher generation share of renewables, the new trend in development of power systems is related to the construction of large scale PV and wind farms with a capacity of hundreds of megawatts. There are many already realized projects of large PV plants like Desert Sunlight Solar Farm and Mount Signal Solar in USA, Solarpark Meuro in Germany and Sunport Delfzijl in the Netherlands.

As a result, large scale integration of renewable energy

This project has received funding from the European Union's Horizon 2020 research and innovation program under grant agreement No 691800 sources to transmission grids have started to make impact on the correct operation of numerical distance relays applied to protect transmission lines. In [3], the impact of renewables on distance protection functioning with attention to false tripping in real grid was reported in.

To get an understanding about the reasons of distance relay unexpected operation, the power electronics that is interfaced to the renewables should be analyzed. A short circuit current contribution of a PV plant was reported in [4], and the conclusion was that the positive and the negative sequence currents are usually suppressed to low values by the inverter control, so that they may influence the relay performance. A PV inverter-enhanced control strategy with a separate positive and negative sequence control was introduced in [5] where the mathematical concept of the control logic was explained in detail. It is worth noting that the disadvantage of this enhanced type of control is the delay in the dynamic response caused by moving average filters. Distance protection performance of overhead lines emanating from full-scale converter-based renewable energy power plants was reported in [6]. Possible solutions for the improvement of distance relay logic were proposed in [7] and investigated by performing simulations in PSCAD environment.

Most of previous studies were based on offline software simulations. Real-time testing can provide better observability of the system performance during testing a physical IED/relay.

The main objective of this research is the testing of actual distance protection of a transmission line radially connected to a large-scale PV source in Real-Time-Digital-Simulator (RTDS). Different scenarios for which the relay may operate incorrectly are examined in detail.

\section{GRID CODE}

For the relay protection study, an important part of TenneT Grid Code [8] is the behavior of a Type-2 generation plant during fault events in the grid. It is required that during fault conditions in the grid, the generation plant is not disconnected from the grid. The disconnection time is defined according to the characteristic shown in Fig. 1. 
When the voltage drop at the point of common coupling is lower than limit line 1 as shown in Fig. 1, a quick disconnection of the generation plant is allowed.

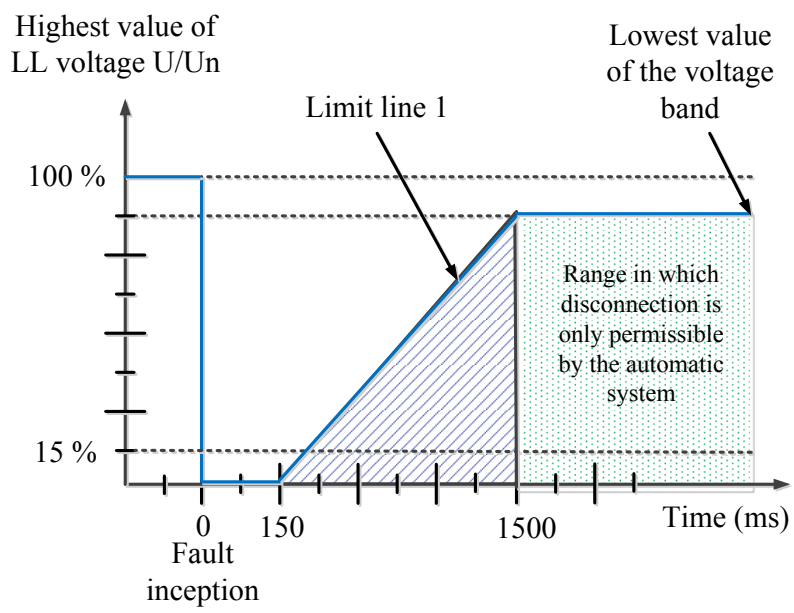

Figure 1. Fault Ride Through characteristic

PV plant must provide a reactive current support during fault conditions; this step is also known as a fault-ride-through requirement. During a fault, when the relative voltage varies more than $10 \%$ of the nominal value, the renewable intermittent source must provide a reactive current according to the red line shown in Fig. 2. When the voltage is $10 \%$ lower than the nominal voltage, the low voltage ride-through (LVRT) characteristic is applied; otherwise high voltage ridethrough characteristic is in use. Renewable intermittent sources must support the grid voltage during all types of faults with not only positive but also a negative short-circuit reactive current injections.

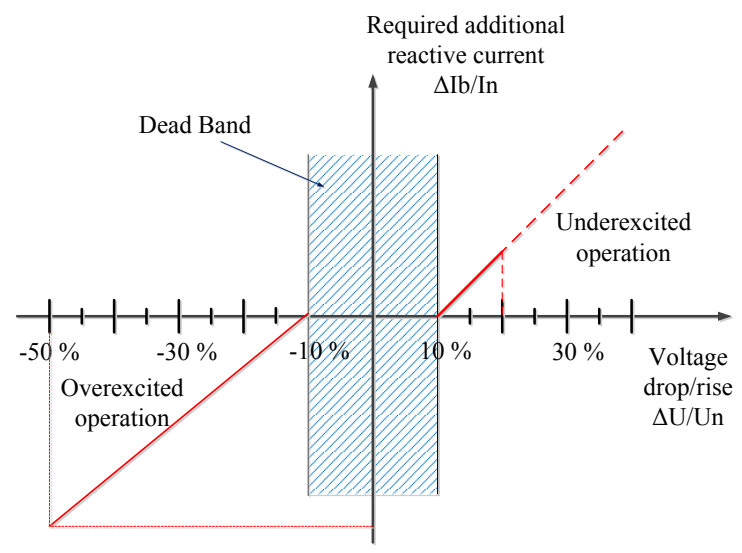

Figure 2. Reactive current support

According to the Tennet Grid Code, at least a distance protection relay must be installed at all grid connection points [8]. In this research, a $400 \mathrm{kV}$ high-voltage transmission line is considered and the following regulations for the protection devices are applied to:

- The PV plant must not self-disconnected during a voltage drop down to $0 \%$ Un with a duration of $\leq 150 \mathrm{~ms}$. When a fault occurred in the interconnection line this must be isolated in less than $150 \mathrm{~ms}$.

- $\quad$ selectivity of the device must be $100 \%$

- single-phase automatic reclosure must be fulfilled on overhead lines with an interval of 1 to 1.2 seconds

The fault ride-through and the reactive current support were implemented in the control of the power electronic converter used for the PV plant, which is based on the positive sequence control.

\section{TEST SYSTEM MODEL}

\section{A. System Topology}

In Fig. 3, the studied network for real time protection testing is shown. The network consists of four synchronous generators and a PV plant as explained in [8]. The system is further enhanced to meet the requirements for the specified analysis of distance protection. The parameters of the lines, loads and generators are taken from [8], and they are all expressed in per unit values.

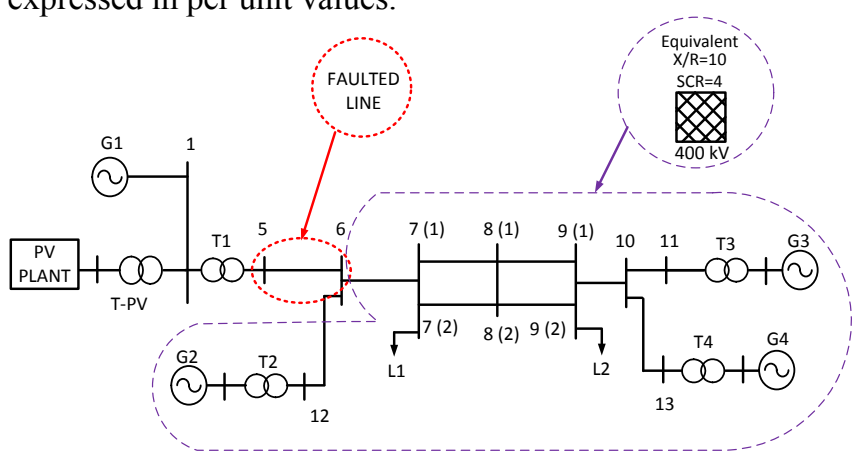

Figure 3. Topology of an enhanced Khundur system

The dynamics produced by the part of the grid on the right from the line under consideration do not compromise the performance of the relays. This part is replaced by an equivalent source, which decreases the use of hardware resources and yield more flexibility in the modeling. The concept of short circuit ratio (SCR) is used to get the same fault current behavior. The values of the impedance magnitude and the impedance angle of the equivalent three-phase voltage source are computed in a way to match the same short circuit contribution; in this case X/R ratio is equal to 10 and the SCR for 100MVA is 4.The PV model is connected to the grid at the same bus as generator G1 at the left side of the line under consideration (see Fig. 3). Two virtual circuit breakers are modeled in order to enable contribution either from the synchronous generator or from the PV connection as shown in Fig. 4.

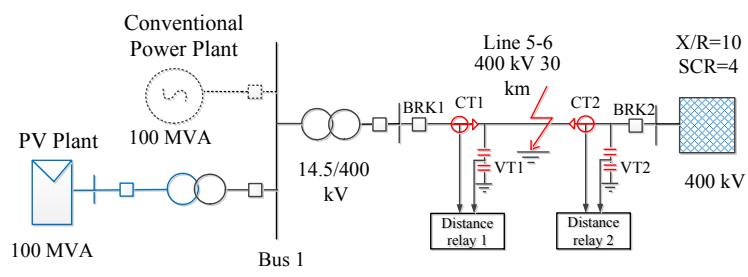

Figure 4. Topology of grid with equivalised source in RSCAD 


\section{B. Hardware-in-the-loop test setup}

The hardware-in-the-loop (HiL) test setup that comprises RTDS and physical equipment consists of several stages. The first stage includes network modeling in RSCAD, the processing of binary signals from the circuit breakers (breaker status signal) and exporting the analog signals from the current and voltage transformers. The second stage is the hardware connection of the distance protection relay. The relays receive voltage and currents from the secondary of the instrument transformers. In this research, the CT and VT are considered as ideal as no saturation is taken into account and the transformer ratios are $2000 / 5 \mathrm{~A}$ and $440 \mathrm{kV} / 110 \mathrm{~V}$ respectively. Finally, the automatic tests are performed by making use of a generic script file that is written in C-type programing language. In this way, the testing process consisting of the preparation of the test environment and changing the requested data for different test scenarios is fully automatized. The data for each test are recorded and saved in an excel file.

A functional block diagram of the HiL tests with the general concept of the setup is shown in Fig. 5. The binary (trip and reclosing) signals generated by the relays are sent to the RTDS simulator.

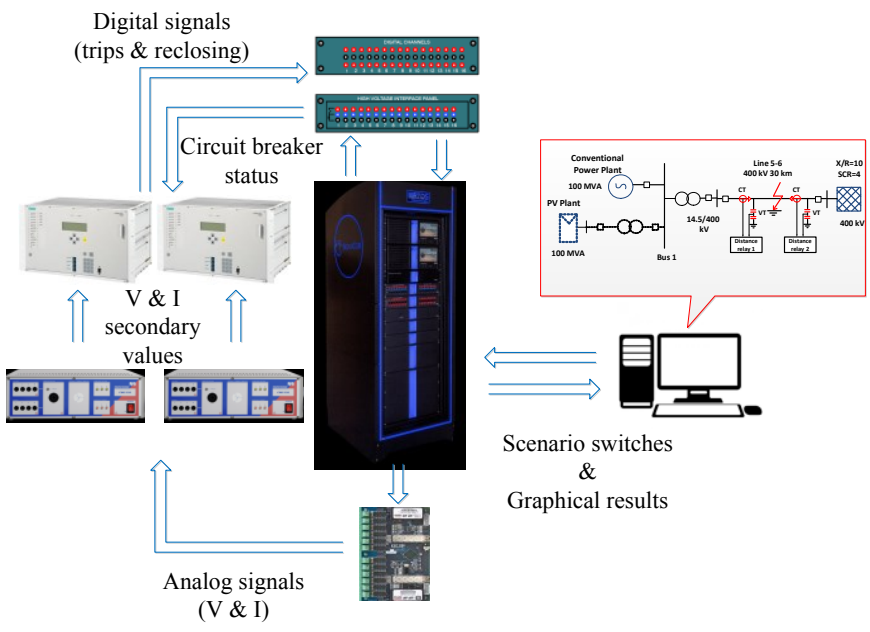

Figure 5. Functional block diagram of the HiL test

The analog signals from the VT and the CT of the two relays are sent out of the hardware in a form of a small voltage that may vary within $\pm 10 \mathrm{~V}$. These voltage signals are amplified by external amplifiers to produce correct secondary voltage and current signals that will be provided to the relay.

The binary signals, namely, the three trip signals for each phase and one reclosing command from the commercial relays are sent back to RSCAD. All these signals have magnitudes not greater than 5V DC. Hence, for the two distance relays connected in a HiL, eight signals are provided and sent to the virtual breakers in the RSCAD model. The breaker status is also sent from RTDS to the relays by a special card that activates a dry contact that closes the DC supplied circuit connected to the physical relay.

The signals of the breakers, which define the breaker state, provide binary outputs for the open and closed position with values equal to zero and one respectively. These data are sent to the relay to indicate whether the relay should trigger or not.

One extra binary signal is responsible for the fault recording action in the commercial numerical relay.

\section{Testing Distance Protection}

The distance protection functionality of two relays is tested in the HiL setup. The recorded oscillographies are saved as COMTRADE files, and trip and reclosing times are stored in a table. By previously explained automatic testing, nearly 100,000 tests for different fault scenarios are performed. Every test is processed in about $12 \mathrm{~s}$ in an average of 5 tests per minute. All the unexpected relay operation cases were identified and classified

\section{A. Test Scenarios}

The test system was designed to simulate the case of gradual replacement of synchronous generators by renewable generators connected by power electronic converters. Originally, the system was fed only by synchronous generators; then the amount is gradually decreased and replaced by PV generation. In order to check all critical fault currents and the impedance reach, three different test scenarios are analyzed:

- The first test case deals with the use of only synchronous generation (G1, G2, G3 and G4 from Fig. 3) every one provides 100 MVA.

- The second test case corresponds to a case where both PV plant and synchronous generators are used at bus 1 with a different share of generation as shown in TABLE I.

- Third case analyzes the performance of the relays where at bus $1,100 \mathrm{MVA}$ is produced only by the PV plant (see Fig. 3 and Fig. 4)

TABLE I. GENERATION OUTPUT FOR DIFFERENT CASES

\begin{tabular}{c|c|c}
\hline \hline $\begin{array}{c}\text { Number of } \\
\text { the case }\end{array}$ & $\begin{array}{c}\text { Synchronous Generator } \\
\text { power output } \\
\text { (MVA) }\end{array}$ & $\begin{array}{c}\text { PV power output } \\
\text { (MVA) }\end{array}$ \\
\hline 1 & 80 & 20 \\
2 & 60 & 40 \\
3 & 50 & 50 \\
4 & 40 & 60 \\
5 & 20 & 80 \\
\hline \hline
\end{tabular}

For all tests, several fault locations along the transmission line 5-6 are denoted. In order to check the selectivity of the protection, the fault locations close to the boundary of the protection zone equal to $80 \%$ of line distance are considered. The simulated fault types are: line-to-ground fault (LG), lineto-line fault (LL), line-to-line-ground fault (LLG) and three phase fault (LLL).

Besides bolted faults (with a resistance of $0.1 \Omega$ ), three different fault resistances are also considered with resistances of $1 \Omega, 10 \Omega$ and $100 \Omega$.

It is worth saying that the relay settings and the Mho impedance are provided by the transmission system operators (TSO). The RLC line positive and zero sequence data are 
$\mathrm{R}^{1}=0.0293 \Omega / \mathrm{km}, \mathrm{X}^{1}=0.3087 \Omega / \mathrm{km} \mathrm{R}^{0}=0.3 \Omega / \mathrm{km}, \mathrm{X}^{0}=0.988$ $\Omega / \mathrm{km}$ and the shunt capacitances are $\mathrm{C}^{1}=0.2664 \mathrm{M} \Omega / \mathrm{km}$ and $\mathrm{C}^{0}=0.4369 \mathrm{M} \Omega / \mathrm{km}$.

\section{B. Simulation Results - Bolted faults}

The bolted faults are faults for which the fault impedance is nearly zero, hence in this case these impedances are set to $0.1 \Omega$. Table I summarizes the most important cases and the relay operation at bus 1 is shown in Table II.

TABLE II. Distance Relay Performance For DifFerent Fault LOCATIONS

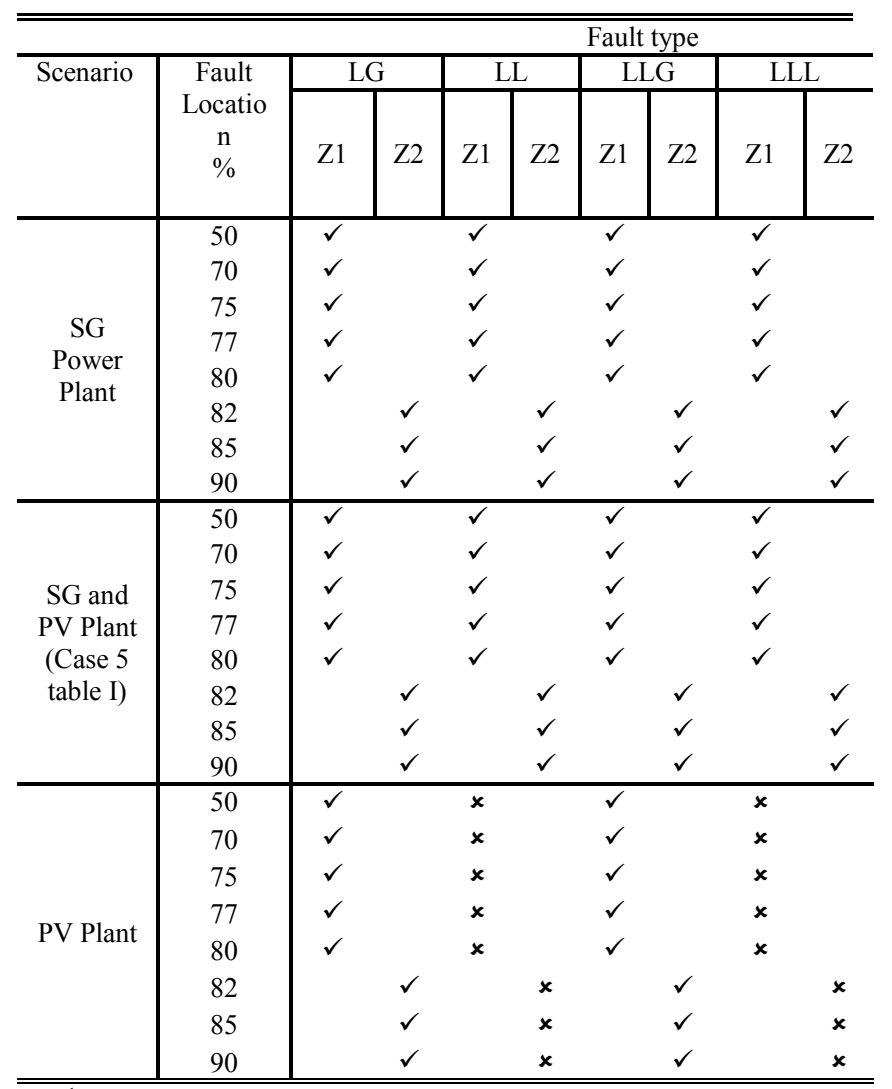

$\checkmark$ Trip command in time

$\times$ No trip

SG Synchronous generator

Z1, Z2 Zone 1 and Zone 2 distance characteristic

The first scenario, which corresponds to $100 \%$ supply by synchronous generators ( $\mathrm{SG})$, proved that the relay at bus 1 operates according to the requirements. This relay is the nearest relay to the $\mathrm{SG}$ and it produces trip commands successfully for any type of fault. Fault locations of $50 \%$, $70 \%, 75 \%, 77 \%$ and $80 \%$ of the line under consideration are in the first zone of the distance protection function. The distance of $80 \%$ (which equals to $24 \mathrm{~km}$ ) is the boundary between Zone 1 and Zone 2. In this case, as seen in Table II faults farther than $80 \%$ of the line was successful detected by the relay which was set to trip after $400 \mathrm{~ms}$ for fault at Zone 2 .

The second test scenario, which is the one with a different share of SG and PV generation (case 5 in Table I) shows that the behavior of the relay is close to the previous scenario where only SGs are connected in the grid. The behavior can be explained by the three parameters; the synchronous steady state reactance $X d$, the transient reactance $X d^{\prime}$ and the subtransient reactance $X d$ " of the synchronous generator. They play a dominant role during the transient process compared to the PV plant control that limits the fault current. $X d$ represents the generator impedance during a steady-state whilst $X d^{\prime}$ 'and $X d^{\prime}$ ' define the generator during a fault condition. In fact, the subtransient component that lasts for nearly two to three periods influences the fault detection of the relay. In this case, it holds that $X d>X d^{\prime}>X d^{\prime \prime}$ ' resulting in a high fault current during the subtransient period. This means that the current injection from the synchronous generator will increase during a fault state shortly after the fault occurs. Generator current injection will prevail even when the PV plant capacity is $80 \%$ of the total generation

The third test scenario, which is a case with only a PV plant connection reveals that the distance protection function fails to operate during LL and LLL faults at any fault location. These types of faults do not involve the ground and therefore the zero sequence components are equal to zero. Moreover, the positive and the negative injected currents are limited by the PV inverter according to the Tennet grid code, which leads to failure of the protective relay to operate. In this case, the fault detection and the phase selection sequences fail as well.

\section{Simulation Results-Impedance faults}

For the second criterion, the fault location is only observed at $80 \%$ of the line length, which is $24 \mathrm{~km}$. For the correct operation, the relay should trigger in the first zone for all cases. The results of these tests are summarized in Table III. The first scenario with a synchronous generator shows that the distance protection function is able to detect the fault for fault impedances up to $1 \Omega$ for any fault type and for fault impedances up to $10 \Omega$ for LG faults. Higher fault impedance makes the impedance trajectory to fall outside the protection zone. The second scenario with a PV connection shows that the protective relay operates correctly when the fault impedance is up to $10 \Omega$ for LG faults and up to $1 \Omega$ for LLG faults. In all other cases, the relay fails to detect the fault.

TABLE III. Distance Relay Performance For Different Fault IMPEDANCES

\begin{tabular}{|c|c|c|c|c|c|c|c|c|c|}
\hline & & & & \multicolumn{6}{|c|}{ Fault type } \\
\hline \multirow[t]{2}{*}{ Scenario } & \multirow{2}{*}{$\begin{array}{c}\text { Fault } \\
\text { imped } \\
\text { ance }\end{array}$} & \multicolumn{2}{|c|}{ LG } & \multicolumn{2}{|c|}{ LL } & \multicolumn{2}{|c|}{ LLG } & \multicolumn{2}{|c|}{ LLL } \\
\hline & & Z1 & Z2 & $\mathrm{Z1}$ & $\mathrm{Z} 2$ & Z1 & Z2 & Z1 & $\mathrm{Z} 2$ \\
\hline \multirow{4}{*}{$\begin{array}{c}\text { SG } \\
\text { Power } \\
\text { Plant }\end{array}$} & 0.1 & $\checkmark$ & & $\checkmark$ & & $\checkmark$ & & $\checkmark$ & \\
\hline & 1 & $\checkmark$ & & $\checkmark$ & & $\checkmark$ & & $\checkmark$ & \\
\hline & 10 & $\checkmark$ & & $x$ & & $x$ & & $x$ & \\
\hline & 100 & $x$ & & $x$ & & $x$ & & $x$ & \\
\hline \multirow{4}{*}{ PV Plant } & 0.1 & $\checkmark$ & & $x$ & & $\checkmark$ & & $x$ & \\
\hline & 1 & $\checkmark$ & & $x$ & & $\checkmark$ & & $x$ & \\
\hline & 10 & $\checkmark$ & & $x$ & & $x$ & & $x$ & \\
\hline & 100 & $x$ & & $x$ & & $x$ & & $x$ & \\
\hline
\end{tabular}

As it is shown in Fig. 6 in case of a PV plant connection, the amplitude of the three phase fault current is close to the load current and relay 1 at the bus side cannot identify the fault. Meanwhile, relay 2, which is located close to the main 
grid successfully operates as the fault current contribution from the network is with much higher value

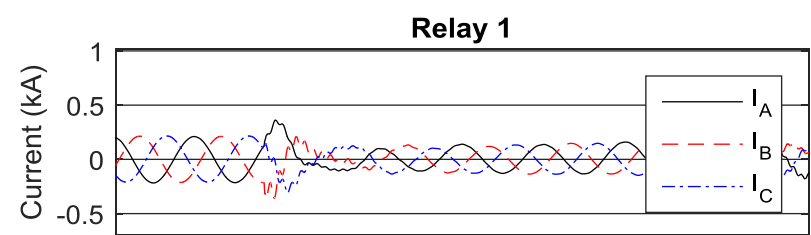

(a)

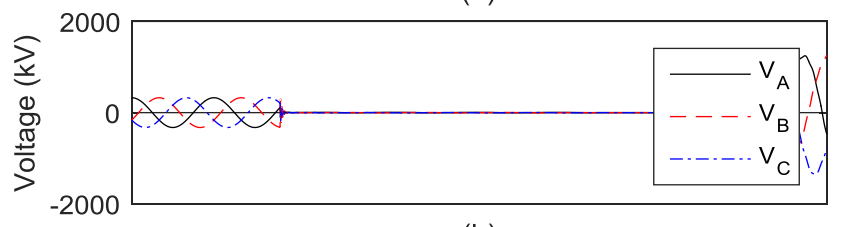

(b)

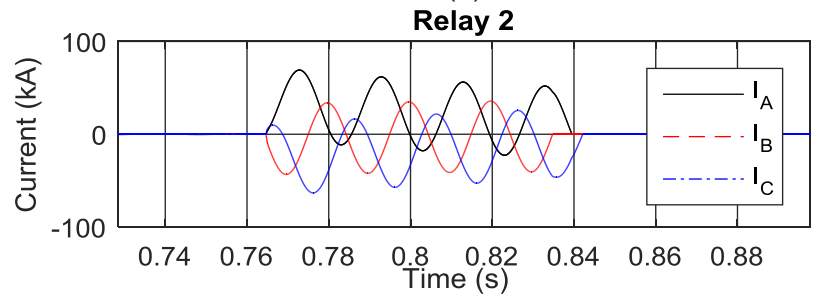

Figure 6. The voltage and the current in relay 1 (bus 5) and the current in relay 2 (bus6) during LLL fault with a PV at bus 1

Fig. 7 shows the impedance trajectories during a LLL bolted fault for impedance between phases $\mathrm{AB}, \mathrm{BC}$ and $\mathrm{CA}$. As it can be seen, the trajectory falls inside the protective zone, however, the relay does not detect the fault. As a result, the sequence of procedures needed for selective clearance of the fault (determination of faulted loop, enabling the impedance and directional verification, trip command, indication of the faulted conductor and supplementary functions) is not accomplished.

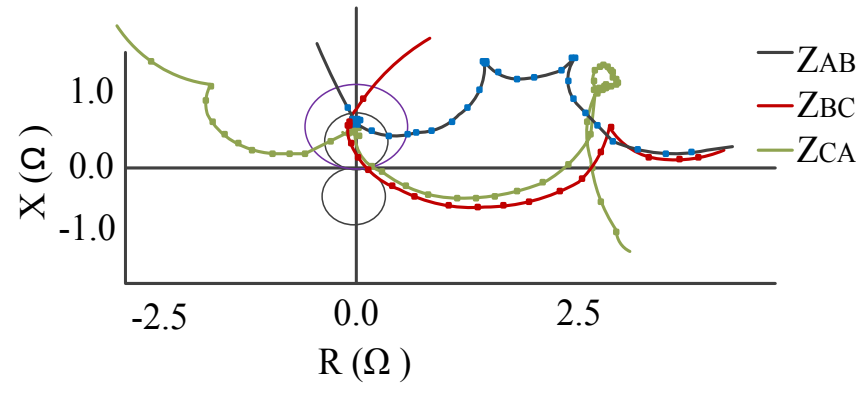

Figure 7. Impedance trajectory per sequence $\mathrm{AB}, \mathrm{BC}$ and $\mathrm{CA}$ at relay 1 during LLL fault with a PV in bus 1

Whilst LLL faults contain only the positive component, LL faults contain positive and negative component, which are controlled by the inverter logic when a PV is connected. Therefore, the first relay does not supply signals to the circuit breaker whereas the second relay connected at bus 6 sends trip and reclose signal in time according to Fig. 8.
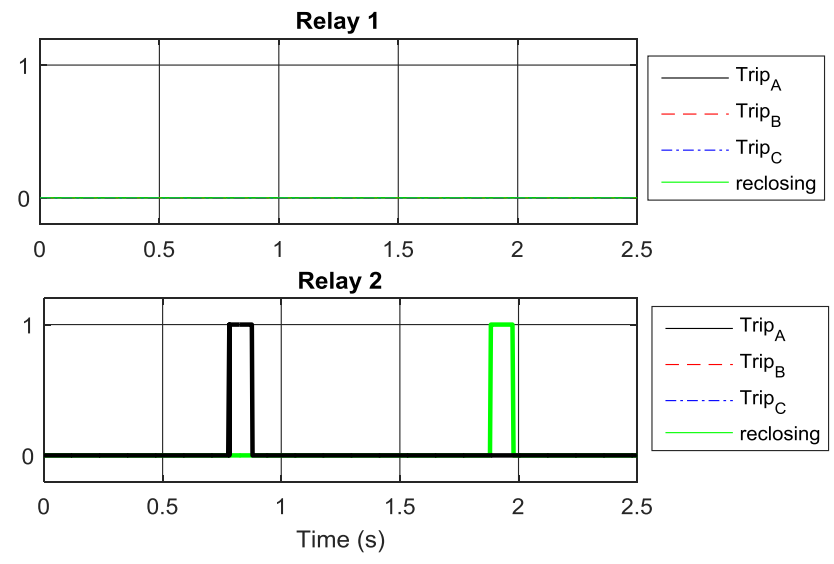

Figure 8. Trip and reclosing signals during LL and LLL fault with PV at bus1

\section{CONCLUSION}

This work has demonstrated the distance relay operation in grid with high penetration of PVs. The tests performed with a simultaneous connection of a PV plant and a conventional generation show correct relay operation due to the high fault currents injected by synchronous generator.

The nuisance tripping is revealed in the relay located close to the PV plant during ungrounded LL and LLL faults. Unexpected behavior is caused by the high dependence of the relay logic from the positive and negative sequence current, which are controlled and limited by the inverter to a value close to the nominal rating of the converter (approximately $1.15 \mathrm{pu}$ ). The current limitation takes place very fast, in the range of milliseconds not allowing the relay to detect the fault. The classical distance protection uses current amplitude as a starting function. As a result, the relay does not trigger even when the impedance trajectory enters the protection zone.

It can be thought that decreasing the current supervision to a low value will produce a successful fault detection. However and in line with TSOs experience, decreasing the current threshold may rise the risk of unexpected trips during switching loads or capacitor banks. Nevertheless, the authors set the overcurrent supervision to the lowest possible and repeated the PV plant cases at TABLE II. Results did not improve, although the relay detect LL faults near its position, the phase detection and directionality were not computed instantaneously delaying the trip for more than $50 \mathrm{~ms}$. The rest of cases did not show a relay enhancement behavior.

A solution should be a distinct starting method. Commercial relays are equipped with the impedance supervision starting function. To compute the impedance between the phase-to-phase loops first the current must be greater than a determined threshold. The overcurrent setting can be set even lower than in overcurrent supervision. However, the proximity of a VSC decrease considerable the current during a fault (see Fig. 6 (b)). In those cases this function results impractical. Other option can be the V/I supervision starting function. Current and/or voltage must exceeds the respective threshold. However, for the cases summarized in this work the current do not trespass the 
threshold and the decrease of the voltage is not always a product of a fault. In this sense this function was not investigated. Some relays use negative sequence supervision for fault detection. Even so, is well known that the VSC suppress or decrease the negative sequence. In that sense the use of negative sequence is not appropriated when a VSC is near the relay.

Results also show that successful detection of LG and LLG faults for PV connection are slower compared to conventional generation case. The average value of delay is around $5 \mathrm{~ms}$. The time lag is created by filters used in the inverter logic. To sum up, the performance of distance protection was compromised in $50 \%$ of cases during PV plant connection to the faulted line.

Although some progress has been made by HiL, further research is required in developing smart algorithms, which will be capable of fault detection and identification to overcome the limitations of the existing functions procedures. Above others, incremental quantities, wavelets detection and Short-Time Fourier based transforms has shown acceptable behavior. These methods should be tested in Real-time based simulation to guarantee results in accuracy and speed

\section{ACKNOWLEDGMENT}

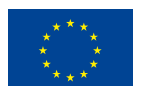

This research was carried out as part of the MIGRATE project. This project has received funding from the European Union's Horizon 2020 research and innovation program under grant agreement No 691800

\section{REFERENCES}

[1] 2030 Climate \& Energy Framework, European Council, 2014 [Online]. Available European Commission. Energy, Climate Change, Environment web site: https://www.consilium.europa.eu/uedocs/cms_data/docs/pressdata/en/e c/145397.pdf

[2] Australia's 2030 Emissions Reduction Target, Australian Government, 2015[Online]. Available:

http://www.environment.gov.au/system/files/resources/f8f337c2-2d584d70-a1fd-acc71254a137/files/factsheet-2030-emissions-reductiontarget.pdf

[3] M. Nagpal, C. Henville, "Impact of Power Electronic Sources on Transmission Line Ground Fault Protection," IEEE Trans. Power Delivery, vol. 3, pp.62-70, Feb. 2018.

[4] A. Hooshyar, M. Azzouz,E.El-Saadany "Distance Protection of Lines Emanating From Full-Scale Converter-Interfaced Renewable Energy Power Plants - Part I: Problem Statement," IEEE Trans. Power Delivery, vol. 30, pp.1770-1780, 2015.

[5] A. Hooshyar, M. Azzouz, E. El-Saadany "Distance Protection of Lines Emanating From Full-Scale Converter-Interfaced Renewable Energy Power Plants - Part II: Solution. Description and Evaluation," IEEE Trans. Power Delivery, vol. 30, pp.1781-1791, 2015

[6] T. Neumann and I. Erlich, "Short Circuit Current Contribution of a Photovoltaic Plant," in Proc. 2012 8th Power Plant and Power System Control Symposium, vol. 45, pp. 343-348, 2012.

[7] M. Mirhosseini, J. Pou, B. Karanayil, V. Agelidis "Positive-and Negative-Sequence Control of Grid-Connected Photovoltaic Systems under Unbalanced Voltage Conditions," in Australasian Universities Power Engineering Conference, pp. 345-361.

[8] Onshore Grid Code, Tennet (GE), 2015 [Online]. Available: https:/www.tennet.eu/fileadmin/user_upload/The_Electricity_Market/ German_Market/Grid_customers/tennet-NAR2015 eng.pdf

[9] P. Kundur, Power System Stability and Control, New York: McGrawHill, 1994 